\title{
Antimicrobial resistance pattern of Gram-negative bacteria of nosocomial origin at a teaching hospital in the Islamic Republic of Iran
}

\author{
H. Khalili, ${ }^{1}$ R. Soltani, ${ }^{1}$ S Afhami, ${ }^{2}$ S. Dashti-Khavidaki ${ }^{1}$ and B. Alijani ${ }^{1}$
}

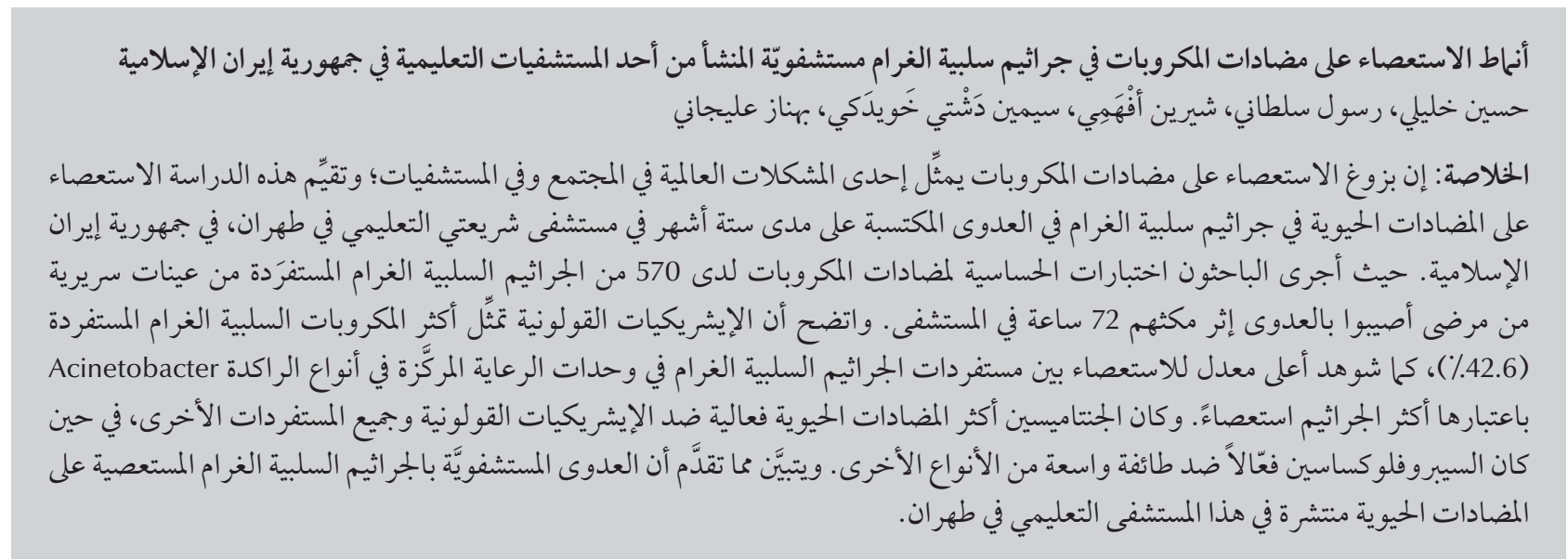

ABSTRACT The emergence of antimicrobial resistance is a global problem in the community and in hospitals. Antibiotic resistance of Gram-negative bacteria from nosocomial infections were evaluated during a 6-month period at Shariati teaching hospital, Tehran, Islamic Republic of Iran. Susceptibility tests were performed on 570 Gram-negative isolates obtained from clinical samples of patients infected after at least 72 hours stay in the hospital. Escherichia coli was the most frequently isolated Gram-negative organism (42.6\%). The highest rate of resistance in Gram-negative isolates was seen in the intensive care unit, with Acinetobacter spp. as the most resistant organisms. Gentamicin was the most effective antibiotic against $E$. coli and all other isolates, while ciprofloxacin was also effective against a wide range of other species. Antibiotic resistant Gram-negative nosocomial infection is prevalent in this teaching hospital in Tehran.

Schéma de résistance aux antimicrobiens des bactéries à Gram négatif d'origine nosocomiale dans un centre hospitalier universitaire de la République islamique d'Iran

RÉSUMÉ La résistance aux antimicrobiens est un problème mondial pour la population comme pour les hôpitaux. La résistance aux antibiotiques des bactéries à Gram négatif responsables d'infections nosocomiales a été évaluée pendant six mois à l'hôpital universitaire Shariati, à Téhéran (République islamique d'Iran). Des épreuves de sensibilité ont été réalisées sur 570 isolats à Gram négatif obtenus à partir d'échantillons cliniques prélevés chez des patients infectés ayant passé au moins 72 heures à I’hôpital. La bactérie à Gram négatif la plus fréquemment isolée était Escherichia coli (42,6 \% des échantillons). Le taux de résistance le plus élevé a été observé dans l'unité de soins intensifs, Acinetobacter spp. étant l'organisme le plus résistant. La gentamicine était l'antibiotique le plus efficace contre $E$. coli et contre tous les autres isolats, mais la ciprofloxacine était également efficace contre un large spectre d'autres espèces. Les infections nosocomiales dues à des bactéries à Gram négatif résistantes aux antibiotiques sont fréquentes dans cet hôpital universitaire de Téhéran.

${ }^{7}$ Department of Clinical Pharmacy, School of Pharmacy; ${ }^{2}$ Department of Infectious Diseases, School of Medicine, Tehran University of Medical Sciences, Tehran, Islamic Republic of Iran (Correspondence to H. Khalili: khalilih@tums.ac.ir).

Received: 23/10/09; accepted: 10/12/09 


\section{Introduction}

Nosocomial or health care-associated infections account for a high morbidity and mortality rate among hospitalized patients [1]. The response to therapy of these infections has changed and antibioticresistance incidencehasincreased at an alarming pace over the past 10 years. About $50 \%-60 \%$ of hospital-acquired infections are caused by resistant strains [2]. A number of factors have been reported that were related to infection with resistant microorganisms, including previous use of antibiotics [3-6], corticosteroid therapy [7], mechanical ventilation [5-7], length of hospital stay [8] and use of invasive devices such as catheters $[9,10]$.

Antimicrobial resistance results in increased morbidity, mortality and costs of treatment. Preventing the emergence and dissemination of resistant organisms is critical for control of hospital infections. Appropriate antimicrobial stewardship that includes optimal selection, dose and duration of treatment, as well as control of antibiotic use, will prevent or slow the emergence of resistance among microorganisms [2].

The prevalence of antimicrobial resistance varies across different settings and studies. Having an awareness of antimicrobial resistance patterns, particularly in hospitals, is crucial for the selection of appropriate antibiotic therapy to improve treatment outcomes, reduce morbidity and mortality, shorten the hospitalization period and consequently reduce the cost of care. The aim of this study was to determine the antimicrobial resistance pattern of Gram-negative bacteria at a teaching hospital in Tehran, Islamic Republic of Iran. Surveillance programmes for regular evaluation of antibiotic resistance patterns are lacking in the Islamic Republic of Iran and this is one of the few comprehensive studies that have been conducted in this area.

\section{Methods}

\section{Setting}

This prospective case-series study was done at Shariati general teaching hospital affiliated to Tehran University of Medical Sciences during December 2007 to May 2008. This is a 500-bed hospital with different medical wards including intensive care unit (ICU), gastroenterology, haematology/oncology and bone marrow transplantation, rheumatology, cardiology, nephrology, neurosurgery, cardiac surgery, general surgery and gynaecology and obstetrics.

\section{Sample}

Biological samples were collected from patients hospitalized in different wards of the hospital who were diagnosed with nosocomial infection. All aerobic Gram-negative isolates collected from patients with at least 72 hours stay in the hospital and without evidence of infection at the time of admission were included in the study. Only 1 isolate per patient per site was included.

For each patient a samples was collected from the most likely site of infection: blood, urine, cerebrospinal fluid (CSF), respiratory tract secretions (collected by bronchoscopy or endotracheal suction), tips of central venous catheters, wound secretions, intra-abdominal abscesses, articular fluid or other clinically relevant sites.

To determine the most important contributing factors to antibiotic drug resistance, demographic data and medical history (including history of invasive procedures and any comorbidity) were recorded for patients with isolates found to be resistant.

\section{Laboratory methods}

Bacterial species were identified by different microbiologic tests, including growth in eosin-methylene-blue and MacConkey media, Gram stain, urease production, $\mathrm{H}_{2} \mathrm{~S}$ production in sulphur-indole motility media, indole production, motility, methyl red test, citrate utilization and decarboxylase production.

Mueller-Hinton agar was used as the growth medium and the antimicrobial susceptibility testing was performed on positive cultures which yielded Gram-negative organisms, using the Kirby-Bauer (disk diffusion) method, in accordance with Clinical and Laboratory Standards Institute (CLSI) guidelines [11]. Susceptibility testing was done for amikacin, gentamicin, tobramycin, ceftazidime, ceftriaxone, cefazolin, cefuroxime, piperacillin, piperacillin/tazobactam, ciprofloxacin, nalidixic acid, and nitrofurantoin.

The results were interpreted after $18-24$ hours of incubation at $35^{\circ} \mathrm{C}$. The zone diameter measured around each disk was measured and classified using CLSI guidelines as susceptible, intermediate or resistant according to the zone size diameters $[11,12]$.

\section{Analysis}

We used SPSS, version 11.5 for data analysis. The results were reported as frequencies. Multivariate analysis was performed to determine variables with independent significant correlations. $P$ values $\leq 0.05$ were considered as statistically significant.

\section{Results}

\section{Organisms isolated}

A total of 570 samples were obtained. Gram-negative organisms were isolated mainly from the urinary tract (45.6\%), blood (21.5\%), and respiratory tract (19.3\%). The remaining isolates (13.1\%) were obtained from wound (6.3\%), CSF (4.8\%) and articular fluid $(2.5 \%)$ or other sources $(2.5 \%)$.

Table 1 shows the organisms isolated from 556 of the samples for which the organism could be identified. Escherichia coli was the most frequently isolated Gram-negative organism (42.8\%), followed by Acinetobacter spp. (17.1\%), Klebsiella spp. (16.5\%), Pseudomonas 
aeruginosa (14.2\%) and Enterobacter spp. (7.6\%). The remaining $1.8 \%$ of isolates included Proteus vulgaris and Morganella morganii.

In terms of the infection site E. coli was the most frequent infecting Gram-negative organism isolated from urine samples and wound secretions, while E. coli, Acinetobacter spp., Klebsiella spp., $P$. aeruginosa and Enterobacter spp., all were isolated from blood specimens in similar proportions (Table 1). Acinetobacter spp. were the most frequently isolated Gram-negative organisms from respiratory tract secretions.

\section{Antibiotic susceptibility}

The overall susceptibility of the isolated Gram-negative organisms to antimicrobial agents was $51.3 \%$ for gentamicin, $47.5 \%$ for ciprofloxacin, $32.0 \%$ for amikacin, $29.7 \%$ for ceftazidime, $22.1 \%$ for ceftriaxone, $13.8 \%$ for nitrofurantoin, , $9.0 \%$ for nalidixic acid, $8.1 \%$ for cefazolin, $7.7 \%$ for tobramycin, $4.3 \%$ for piperacillin/ tazobactam, $2.5 \%$ for piperacillin and $1.3 \%$ for cefuroxime (Table 2). Gentamicin was the most effective antibiotic against E. coli, while ciprofloxacin was also effective against a wide range of other Gram-negative bacteria isolated. The least effective agent for all isolated Gram-negative microorganisms was cefuroxime.

\section{Patient evaluation}

A total of 60 patients with resistant isolates were evaluated. Among the studied patients, $58.3 \%$ (35 cases) were male. The mean age of the patients was 49.5 (SD 22.3) years.

Of the patients $35.0 \%$ had history of recent hospitalization (for at least 48 hours, within the last 3 months) and $40.0 \%$ of them had a history of a recent surgery (within the previous 3 months). Concerning the presence of comorbidity, chronic heart failure (28.3\%), diabetes (21.7\%), chronic renal failure $(21.7 \%)$ and impaired immune system function due to long-term corticosteroid use (18.3\%) were the most common comorbid conditions reported. Additionally, $16.7 \%$ of patients were receiving total parenteral nutrition during hospitalization.

Of these potential predisposing factors, history of recent hospitalization ( $\mathrm{OR}=3.9,95 \% \mathrm{CI}: 1.8-14.5)$, history of recent surgery $(\mathrm{OR}=2.3,95 \% \mathrm{CI}: 1.1-8.6)$ and long-term corticosteroid therapy $(\mathrm{OR}=1.9,95 \% \mathrm{CI}: 1.2-6.9)$ were significantly associated with presence of bacterial resistant isolates (Table 3 ).

\section{Discussion}

Nosocomial infections are a serious threat to hospitalized patients. The emerging problem of antimicrobial resistance further complicates the issue because most deaths associated with nosocomial infection are caused by antibiotic-resistant microorganisms $[1,2]$.

In our study, based on the culture sources, the most frequent nosocomial Gram-negative infections were urinary tract infection (45.6\%), followed by bacteraemia (21.5\%) and pneumonia (19.3\%). In a recent study in ICU patients of 2 other teaching

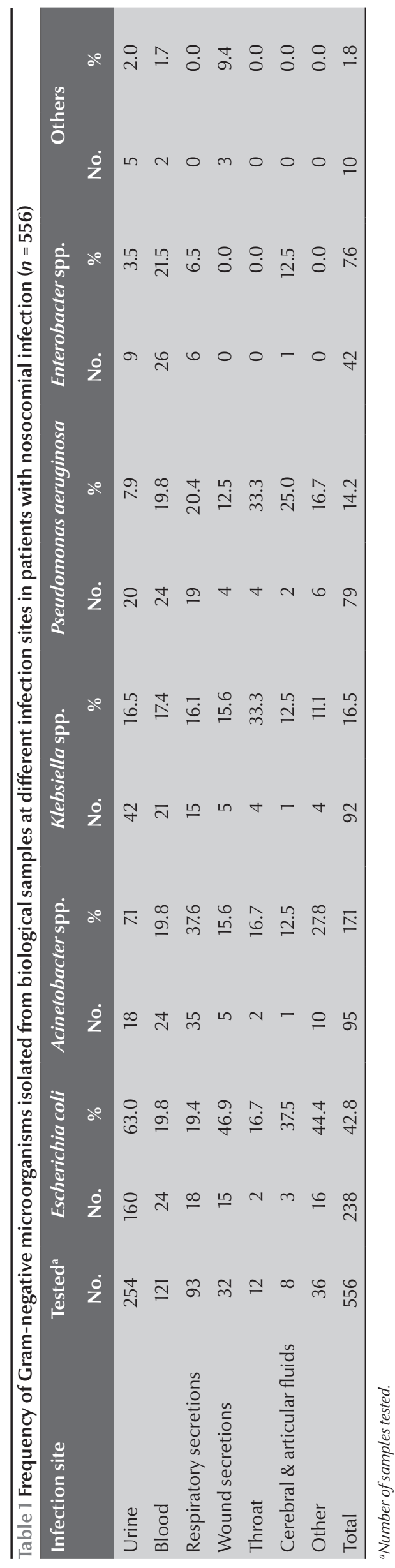




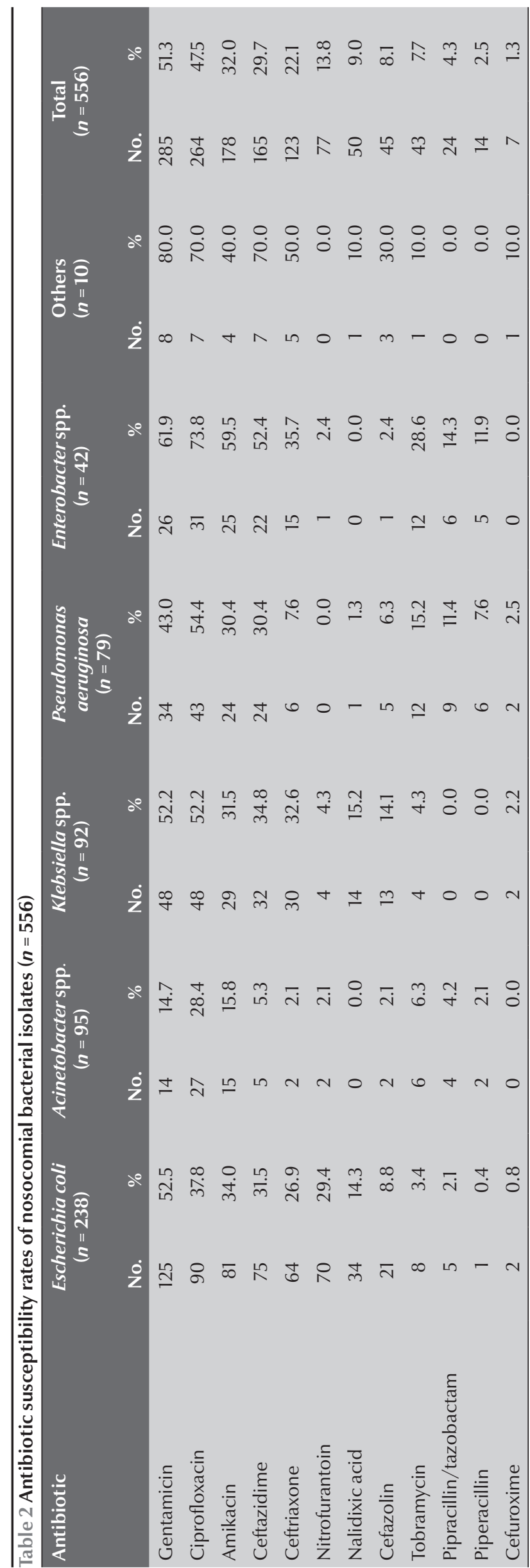

hospitals of Tehran, Islamic Republic of Iran (Sina and Imam Khomeini hospitals) performed by Hadadi et al., septicaemia (33.0\%) and pneumonia (29.5\%) were the most frequent nosocomial infections [13]. In the study of Vesal et al. performed on Gram-negative isolates of ICU patients at Shariati hospital during a 6-month period from October 2003 to April 2004, pneumonia was the most frequent nosocomial infection [14]. These slightly different results could be explained by the difference in the patient population of these studies; our study included patients from all medical wards whereas other studies were of ICU patients only.

In our study, E. coli was the most frequent isolate in urinary tract infection and a major isolate in bacteraemia. This shows that urosepsis is a major cause of nosocomial infections.

Acinetobacter spp. and $P$. aeruginosa were the most frequently isolated Gram-negative bacteria from the respiratory tract in the current study. This is in agreement with the study of Vesal et al. that reported Acinetobacter as the most frequent isolated species [14]. In contrast, in the study of Hadadi et al. Klebsiella spp. and P. aeruginosa were the most frequent isolates from respiratory tract specimens [13]. Therefore, it can be concluded that Acinetobacter spp. and $P$. aeruginosa are major pathogens responsible for nosocomial pneumonia.

In the current study, Acinetobacter spp. were the most antibiotic-resistant Gram-negative microorganisms. This is in agreement with a study in Chicago in 2004 that reported a high resistance rate of Acinetobacter spp. to all commonly used antibiotics with a resultant high morbidity and mortality rate [15]. Similar results have been reported in a Spanish study in 2004 [16].

In our study, Acinetobacter spp. was both the most frequently isolated agent from the respiratory tract and the most resistant of all isolates. This highlights the need for new effective antimicrobial agents and strategies to prevent and treat common respiratory tract infections such as hospital-acquired pneumonia.

Some other studies have reported $P$. aeruginosa as the most resistant organism $[17,18]$. This is consistent with our study that showed this pathogen as the second most resistant organism. In the current study, the most effective antibiotic against this pathogen was ciprofloxacin with a susceptibility of $54.4 \%$, while in the study of Hadadi et al. the susceptibility of $P$. aeruginosa to this antibiotic was $33.3 \%[13]$.

Although ciprofloxacin, amikacin and gentamicin were the most effective agents against Gram-negative isolates in our research, nevertheless between 49\%-68\% of all isolates were resistant to these antibiotics. 


\begin{tabular}{|c|c|c|c|c|c|c|}
\hline \multirow[t]{2}{*}{ Variable } & \multicolumn{2}{|c|}{ Resistant } & \multicolumn{2}{|c|}{ Not resistant } & \multirow[t]{2}{*}{ Crude OR $(95 \% \mathrm{CI})$} & \multirow[t]{2}{*}{$P$-value } \\
\hline & No. & $\%$ & No. & $\%$ & & \\
\hline \multicolumn{7}{|l|}{ Age group (years) } \\
\hline$<50$ & 24 & 40.0 & 210 & 42.3 & $1.1(0.8-1.6)$ & 0.33 \\
\hline $50-59$ & 20 & 33.3 & 160 & 32.3 & $0.8(0.3-1.2)$ & 0.51 \\
\hline $60-69$ & 12 & 16.7 & 100 & 20.2 & $1.3(0.5-1.5)$ & 0.30 \\
\hline $70+$ & 4 & 6.6 & 26 & 5.3 & $0.9(0.4-1.5)$ & 0.44 \\
\hline \multicolumn{7}{|l|}{ Sex } \\
\hline Male & 35 & 58.3 & 256 & 51.6 & $0.4(0.1-1.4)$ & 0.31 \\
\hline Female & 25 & 41.7 & 240 & 48.8 & $1.3(0.4-1.9)$ & 0.81 \\
\hline \multicolumn{7}{|l|}{ Clinical history } \\
\hline History of recent hospitalization & 21 & 35.0 & 63 & 13.8 & $3.9(1.8-14.5)$ & 0.003 \\
\hline History of recent surgery & 24 & 40.0 & 73 & 16.0 & $2.3(1.1-8.6)$ & 0.01 \\
\hline Long-term corticosteroid therapy & 11 & 18.3 & 57 & 12.5 & $1.9(1.2-6.9)$ & 0.004 \\
\hline
\end{tabular}

$O R=$ odds ratio $; C l=$ confidence interval.

As expected, cefuroxime was the least effective agent against isolates and its empirical use in nosocomial Gramnegative infections should therefore be avoided.

Our study detected several factors, including history of surgery and hospitalization, and some comorbidities, including heart failure, diabetes, chronic renal failure and long-term corticosteroid use, as possible contributing risk factors to antimicrobial resistance in patients from whom resistant organisms were isolated. However, a larger study with a larger sample is needed to confirm these data.

In conclusion, the rate of antimicrobial resistance is high among nosocomial Gram-negative bacteria in this hospital, complicating the decision to start appropriate antibiotics to manage nosocomial infections empirically. A surveillance system is recommended to record and report the antimicrobial resistance pattern of hospital isolates periodically, as this pattern changes continually over time.

\section{Acknowledgements}

We are grateful to the staff of the microbiology laboratory of Shariati hospital for their participation in this work.

\section{References}

1. Vincet JL et al. The prevalence of nosocomial infection in intensive care units in Europe. The result of the European Prevalence of Infection in Intensive Care (EPIC) study. Journal of the American Medical Association, 1995, 274:639-644.

2. Shlaes DM et al. Society for Healthcare Epidemiology of America and Infectious Diseases Society of America joint committee on the prevention of antimicrobial resistance: Guidelines for the prevention of antimicrobial resistance in hospitals. Infection Control and Hospital Epidemiology, 1997, 18:275-291.

3. Fagon JY et al. Nosocomial pneumonia in patients receiving continuous mechanical ventilation. Prospective analysis of 52 episodes with use of a protected specimen brush and quantitative culture techniques. American Review of Respiratory Disease, 1989, 139:877-884.

4. Husni RN et al. Risk factors for an outbreak of multi-drug-resistant Acinetobacter nosocomial pneumonia among intubated patients. Chest, 1999, 115:1378-1382.

5. Rello J et al. Risk factors for infection by Pseudomonas aeruginosa in patients with ventilator-associated pneumonia. Intensive Care Medicine, 1994, 20:193-198.

6. Trouillet JL et al. Ventilator-associated pneumonia caused by potentially drug-resistant bacteria. American Journal of Respiratory and Critical Care Medicine, 1998, 157:531-539.
7. Ibelings MM, Bruining HA. Methicillin-resistant Staphylococcus aureus: acquisition and risk of death in patients in the intensive care unit. European Journal of Surgery, 1998, 164:411-418.

8. Bonten MJ et al. External sources of vancomycin-resistant enterococci for intensive care units. Critical Care Medicine, 1998 26:2001-2004

9. Richards MJ et al. Nosocomial infections in medical intensive care units in the United States. National Nosocomial Infections Surveillance System Critical Care Medicine, 1999, 27:887-892.

10. Kollef $\mathrm{MH}$ et al. The impact of nosocomial infections on patient outcomes following cardiac surgery. Chest, 1997, 112:666-675.

11. Performance standards for antimicrobial susceptibility testing: nineteenth informational supplement M100-S19. Wayne, Pennsylvania, Clinical and Laboratory Standards Institute, 2009.

12. Bauer AW et al. Antibiotic susceptibility testing by a standardized single disk method. American Journal of Clinical Pathology, 1996, 45:493-496.

13. Hadadi $A$ et al. Antimicrobial resistance pattern of Gramnegative bacilli of nosocomial origin at 2 university hospitals in Iran. Diagnostic Microbiology and Infectious Disease, 2008, 60:301-305. 
14. Gh V et al. Evaluation of antimicrobial resistance among Gramnegative isolates collected from intensive care units and reliability of routine disc susceptibility tests at a teaching hospital in Tehran. Iranian Journal of Pharmaceutical Research, 2006, 2:89-100.

15. Jain R, Danziger LH. Multidrug-resistant Acinetobacter infections: an emerging challenge to clinicians. Annals of Pharmacotherapy, 2004, 38:1449-1459.

16. Iglesias de Sena $\mathrm{H}$ et al. Epidemiological study and effect on antimicrobial use in the genus Acinetobacter in a university hospital. Revista Espanola de Quimioterapia, 2004, 17:177-183.
17. Rhomberg PR et al.MYSTIC Programme Study Group. Antimicrobial resistance rates and clonality results from the Meropenem Yearly Susceptibility Test Information Collection (MYSTIC) programme: report of year five (2003). Diagnostic Microbiology and Infectious Disease, 2004, 49:273-281.

18. Shamsuzzaman AK et al. Pattern of aerobic bacteria with their drug susceptibility of surgical inpatients. Mymensingh Medical Journal, 2003, 12:98-103.

\section{Communicable diseases in the Eastern Mediterranean Region. Prevention and control 2005-2009}

In the World Health Organization (WHO) Eastern Mediterranean Region, communicable diseases are among the major causes of mortality and morbidity and pose major impediments to social and economic wellbeing. Communicable diseases in the Eastern Mediterranean Region. Prevention and control 2005-2009 provides an overview of the situation of communicable diseases, the progress made by countries with support from WHO and partners, and the key challenges for disease prevention and control during the period 2005-2009. The report describes the status in relation to 6 visions for communicable diseases prevention and control in the Region: Elimination and eradication of specific diseases; Expanding disease-free areas; Providing a safe vaccine for every childhood disease for every child; Curbing the HIV/ AIDS epidemic; Halving the burden of tuberculosis - working towards elimination and; Containing new and reemerging disease threats.

This full text of this publication can be accessed online at: http://www.emro.who.int/dsaf/dsa1212.pdf 